\title{
C2 Alcohol Oxidation Boosted by Trimetallic PtPbBi Hexagonal Nanoplates
}

ZhiQiang Zhul, Feng Liu ${ }^{1}$, Jinchen Fan ${ }^{1,2}$, QiaoXia Li ${ }^{1,2 *}$, Yulin Min ${ }^{1,2}$, Qunjie Xu ${ }^{1,2}$

${ }^{1 .}$ Shanghai Key Laboratory of Materials Protection and Advanced Materials in

Electric Power, College of Environmental and Chemical Engineering, Shanghai

University of Electric Power, Yangpu District, 2588 Changyang Road, Shanghai, 200090, China

2.Shanghai Institute of Pollution Control and Ecological Security, Shanghai 200090, China

*E-mail: liqiaoxia@shiep.edu.cn 


\section{EXPERIMENTAL SETION}

\section{Reagents and chemicals}

Platinum (II) acetylacetonate $\left(\mathrm{Pt}(\mathrm{acac})_{2}, 97 \%\right)$, lead (II) acetylacetonate $\left(\mathrm{Pb}(\mathrm{acac})_{2}\right.$, 95\%), bismuth (III) nitrate pentahydrate $\left(\mathrm{Bi}\left(\mathrm{NO}_{3}\right)_{3} \cdot 5 \mathrm{H}_{2} \mathrm{O}, 99 \%\right.$ ), oleylamine (oAm, 80 90\%) and octadecene (ODE, 90\%) were purchased from Aladdin. Cetyl trimethyl ammonium bromide (CTAB, 99\%), ascorbic acid (AA, 99\%), ethanol (GR, 99.8\%), ethylene glycol (GR, 99\%), hexane (AR, 99.5\%) and sulfuric acid $\left(\mathrm{H}_{2} \mathrm{SO}_{4}, 98 \%\right)$ were purchased from Sinopharm Chemicals Reagent Co. Ltd., China. Vulcan XC-72R carbon black was obtained from Cabot Co. Nafion (5 wt.\%) was bought from DuPont. The commercial Pt/C catalyst (20 wt.\%) was obtained from Johnson Matthey (JM). All reagents were used without further purification and all solutions were freshly prepared with ultrapure water $(18.2 \mathrm{M} \Omega / \mathrm{cm})$.

\section{Synthesis of catalyst}

Synthesis of PtPbBi hexagonal nanoplates (HNPs): In the typical synthesis of PtPbBi HNP-1, $20.50 \mathrm{mg}$ of $\mathrm{Pt}(\mathrm{acac})_{2}, 9.95 \mathrm{mg}$ of $\mathrm{Pb}(\mathrm{acac})_{2}, 18.88 \mathrm{mg}$ of $\mathrm{Bi}\left(\mathrm{NO}_{3}\right)_{3} \cdot 5 \mathrm{H}_{2} \mathrm{O}, 100$ $\mathrm{mg}$ of $\mathrm{AA}$ and $200 \mathrm{mg}$ of $\mathrm{CTAB}$ were added to a $10 \mathrm{~mL}$ mixed solution $(5 \mathrm{~mL}$ of oAm and $5 \mathrm{~mL}$ of ODE) and ultrasonically oscillated until fully dissolved. The mixed solution was then kept in an oil bath at $210{ }^{\circ} \mathrm{C}$ for $30 \mathrm{~min}$. After being cooled down to room temperature, the products were precipitated with an ethanol/hexane mixture via centrifugation (9000 rpm for $5 \mathrm{~min}$ ). The mixed solution of ethanol and cyclohexane was used to wash the products after centrifugation several times and dispersed in the ethanol solution to obtain a standby solution. An appropriate amount of activated 
carbon (Vulcan XC-72R carbon) was added to the standby solution to reach a $\mathrm{Pt}$ fraction of $20 \mathrm{wt} . \%$. The solution was sonicated for $1 \mathrm{~h}$ and stirred for $3 \mathrm{~h}$ so that the PtPbBi HNPs were evenly loaded onto the carbon powder. After stirring, it was vacuum filtered and dried in a vacuum oven at $60{ }^{\circ} \mathrm{C}$ for $12 \mathrm{~h}$.

The synthesis processes of PtPbBi HNP-2 and PtPbBi HNP-3 were similar to that of $\mathrm{PtPbBi} \mathrm{HNP}-1$, except that precursors of $20.50 \mathrm{mg}$ of $\mathrm{Pt}(\mathrm{acac})_{2}, 9.95 \mathrm{mg}$ of $\mathrm{Pb}(\mathrm{acac})_{2}$ and $16.52 \mathrm{mg}$ of $\mathrm{Bi}\left(\mathrm{NO}_{3}\right)_{3} \cdot 5 \mathrm{H}_{2} \mathrm{O}$, and $20.50 \mathrm{mg}$ of $\mathrm{Pt}(\mathrm{acac})_{2}, 9.95 \mathrm{mg}$ of $\mathrm{Pb}(\mathrm{acac})_{2}$ and $14.16 \mathrm{mg}$ of $\mathrm{Bi}\left(\mathrm{NO}_{3}\right)_{3} \cdot 5 \mathrm{H}_{2} \mathrm{O}$ were used, respectively.

The synthesis of $\mathrm{PtPb} / \mathrm{C}$ and $\mathrm{PtBi} / \mathrm{C}$ were also similar to the synthesis of $\mathrm{PtPbBi} \mathrm{HNP}$ 1, except that $20.50 \mathrm{mg}$ of $\mathrm{Pt}(\mathrm{acac})_{2}$ and $9.95 \mathrm{mg}$ of $\mathrm{Pb}(\mathrm{acac})_{2}$, and $20.50 \mathrm{mg}$ of $\mathrm{Pt}(\mathrm{acac})_{2}$ and $18.88 \mathrm{mg}$ of $\mathrm{Bi}\left(\mathrm{NO}_{3}\right)_{3} \cdot 5 \mathrm{H}_{2} \mathrm{O}$ were used as precursors, respectively.

\section{Characterization}

Transmission electron microscopy (TEM) studies were carried out with a JEOL JEM2100F electron microscope. Atomic force microscopy (AFM) measurements were conducted in an Agilent 5500AFM with tapping mode. X-ray diffraction (XRD) patterns were acquired with a Bruker D8 advance diffractometer. X-ray photoelectron spectroscopy (XPS) measurements were obtained on an ESCALAB 250 photoelectron spectrometer (Thermo VG Scientific, USA) by using Al Ka $(1486.6 \mathrm{eV})$ as the X-ray source. Inductively coupled plasma-atomic emission spectroscopy (ICP-AES) measurements were carried out with a Shimadzu ICPS-7510 instrument.

\section{Electrochemical measurements}


All electrochemical tests were carried out using a CHI660E electrochemical workstation (Shanghai Chenhua Instrument Corporation, China) in a standard threecompartment electrochemical cell at room temperature. Glassy carbon $(\mathrm{GC}, 3 \mathrm{~mm}$ in diameter) covered with catalyst acted as a working electrode, a saturated calomel electrode (SCE) was used as a reference electrode and a Pt net worked as a counter electrode. Before each experiment, the GC electrode was mechanically polished with 0.5 and $0.3 \mu \mathrm{m}$ alumina polishing powders to obtain a mirror surface. The polished electrode was then rinsed with ultrapure water and perform ultrasonic treatment in ethanol and water, respectively. The ink was made with $2.0 \mathrm{mg}$ of the as-obtained catalyst powder dispersed in $1.12 \mathrm{~mL}$ of a Milli-Q water, ethanol and 5 wt.\% Nafion ${ }^{\circledR}$ mixing solution $(\mathrm{v} / \mathrm{v} / \mathrm{v}=2: 1: 0.2)$ and then ultrasonicated for $45 \mathrm{~min}$ in an ice bath to form a uniform ink. Catalyst ink $(5.6 \mu \mathrm{L})$ was dripped on the $\mathrm{GC}$ and dried at room temperature. The Pt loading of all samples was kept at $20 \mu \mathrm{g} \mathrm{cm}^{-2}$ (determined by ICPAES). For the cyclic voltammetry $(\mathrm{CV})$ curves, the electrochemical reactions were carried out in $\mathrm{N}_{2}$ saturated $1 \mathrm{M} \mathrm{NaOH}$ at a scan rate of $50 \mathrm{mV} \mathrm{s}^{-1}$. The electrochemical EOR and EGOR reactions were conducted by $\mathrm{CV}$ in $\mathrm{N}_{2}$ saturated $1 \mathrm{M} \mathrm{NaOH}$ and $1 \mathrm{M}$ ethanol or $1 \mathrm{M}$ ethylene glycol at a scan rate of $50 \mathrm{mV} \mathrm{s}^{-1}$, respectively.

The long-term stability of the as-prepared catalysts was assessed by chronoamperometric (CA) measurements at $-0.2 \mathrm{~V}$ vs. SCE $\left(-0.3 \mathrm{~V}\right.$ vs. SCE) in $\mathrm{N}_{2}$ saturated $1 \mathrm{M} \mathrm{NaOH}$ solution containing $1 \mathrm{M}$ ethanol (or $1 \mathrm{M}$ ethylene glycol). In the $\mathrm{CO}$ stripping experiment, the working electrode was held in a $0.5 \mathrm{M} \mathrm{H}_{2} \mathrm{SO}_{4}$ solution for 15 min with high-purity gaseous $\mathrm{CO}$ bubbled and then switched to $\mathrm{N}_{2}$ for 15 min to 
remove the remaining $\mathrm{CO}$ dissolved in the $\mathrm{H}_{2} \mathrm{SO}_{4}$ solution. Finally, two complete cycles of CO-stripping voltammograms were recorded from -0.20 to $1.00 \mathrm{~V}$ at a scan rate of $50 \mathrm{mV} \mathrm{s}^{-1}$. For comparison, commercial Pt/C (20 wt.\% of Pt nanoparticles supported on Vulcan XC-72 carbon, JM) was employed as a reference to our system. All electrochemical experiments were carried out at room temperature. 


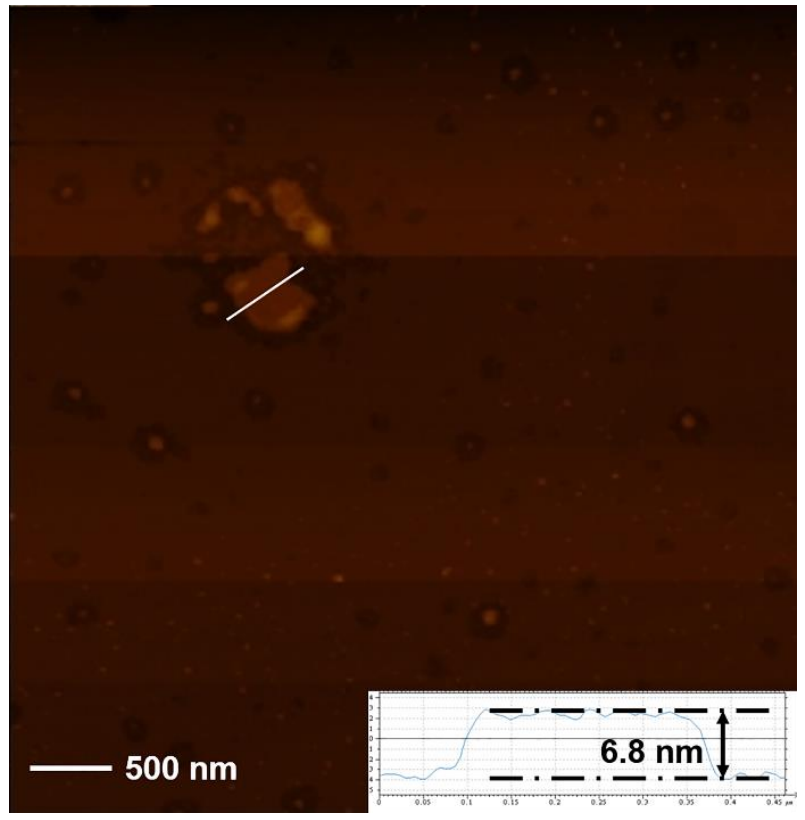

Figure S1. AFM image of the PtPbBi HNPs.

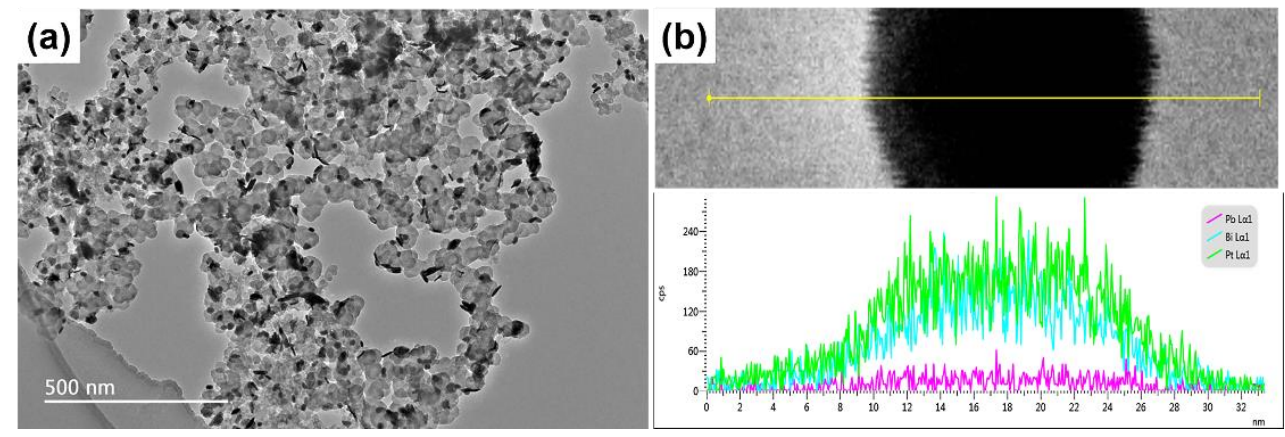

Figure S2. (a) TEM images of PtPbBi HNP-1 (b) EDS line scan of the single PtPbBi hexagonal nanoplates. 

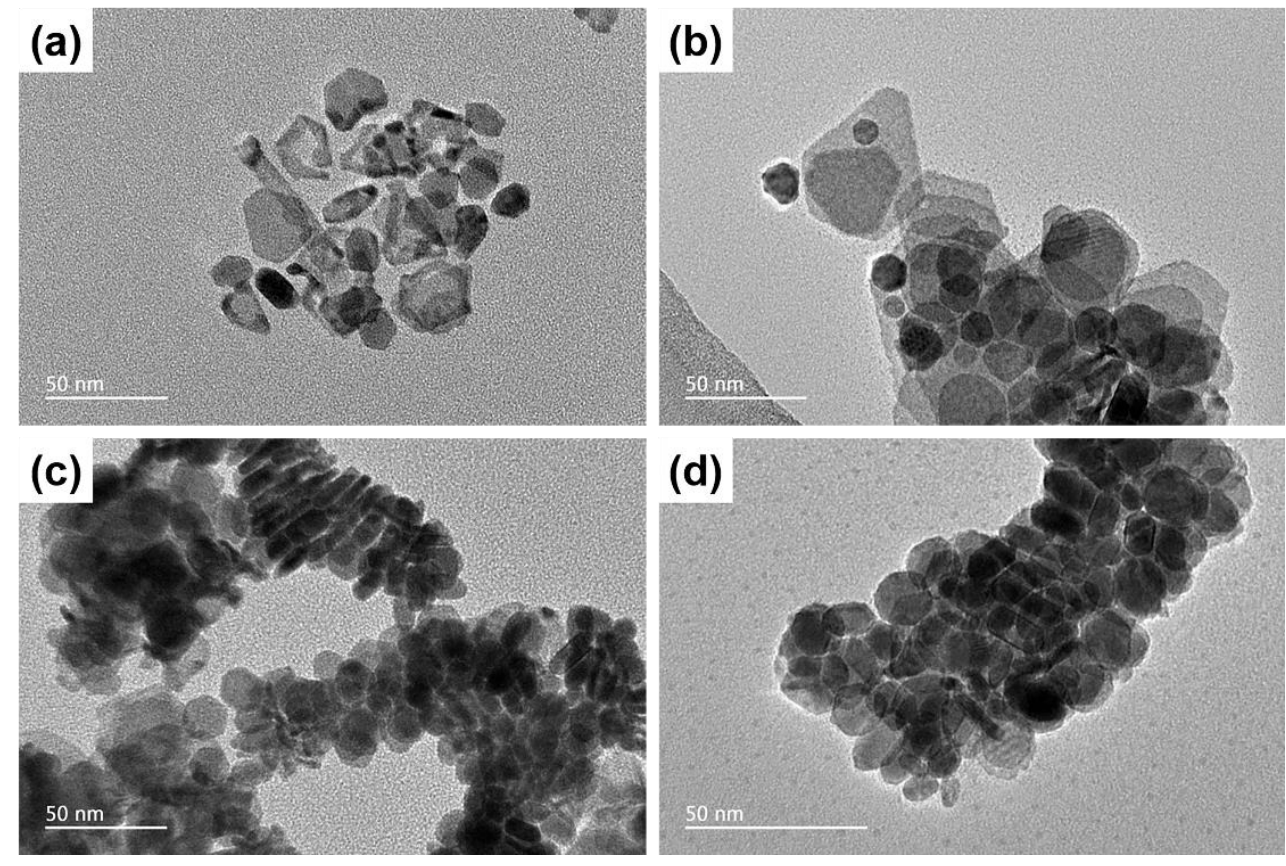

Figure S3. TEM images of the PtPbBi HNP-1 prepared using the standard procedure at different reaction times: (a) 10, (b) 20, (c) 40, and (d) $50 \mathrm{~min}$.

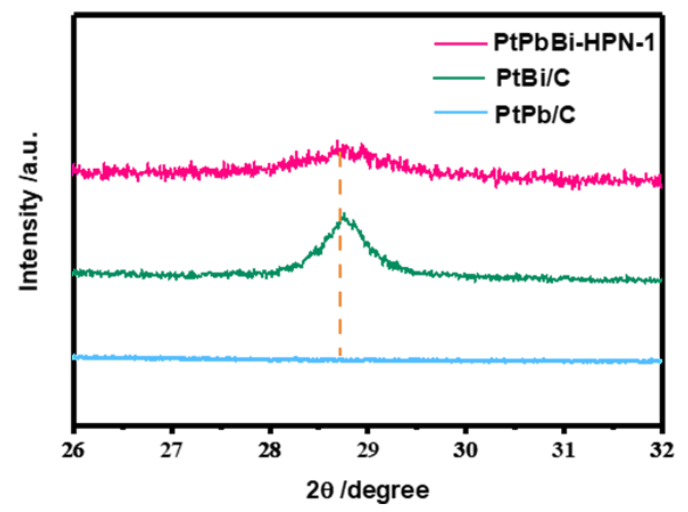

Figure S4. The XRD spectra of $\mathrm{PtPbBi}-\mathrm{HPN}-1, \mathrm{PtBi} / \mathrm{C}$ and $\mathrm{PtPb} / \mathrm{C}$ samples were amplified in the range of $2 \Theta=36^{\circ}-44^{\circ}$. 

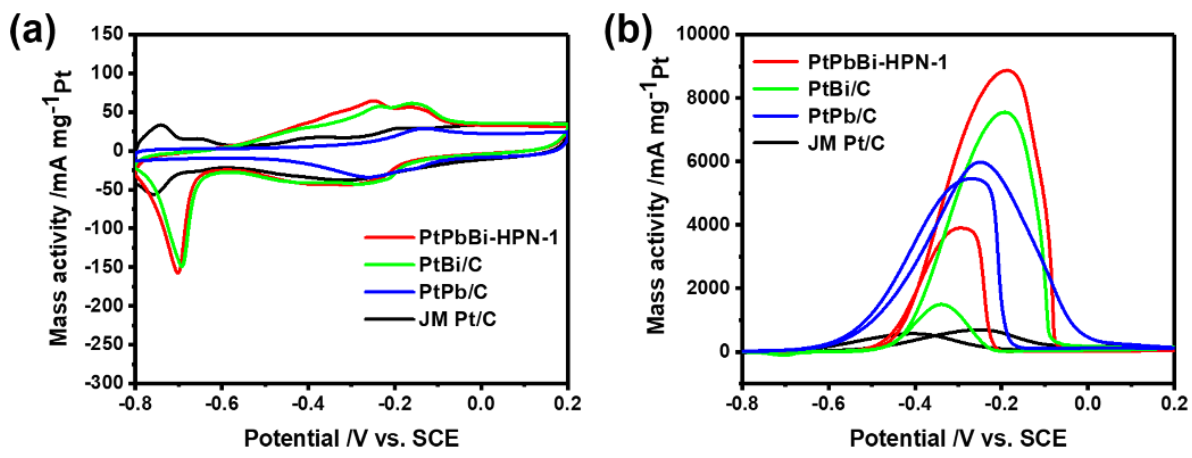

Figure S5. CV curves obtained from $\mathrm{PtPbBi}-\mathrm{HPN}-1, \mathrm{PtBi} / \mathrm{C}, \mathrm{PtPb} / \mathrm{C}$ and $\mathrm{JM} \mathrm{Pt} / \mathrm{C}$ in $\mathrm{N}_{2}$-saturated (a) $1 \mathrm{M} \mathrm{NaOH}$ and (b) $1 \mathrm{M} \mathrm{NaOH}$ solution containing $1 \mathrm{M}$ ethanol solution at a scan rate of $50 \mathrm{mV} \mathrm{s}^{-1}$.

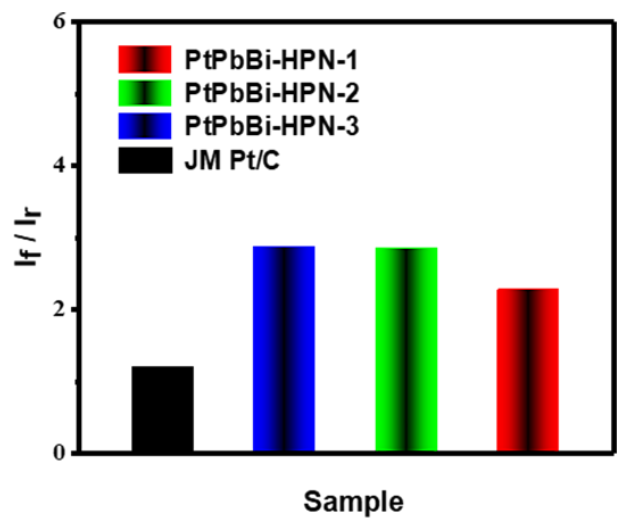

Figure S6. PtPbBi hexagonal nanoplates and $\mathrm{JM} \mathrm{Pt} / \mathrm{C}$ of the ratio between the intensity of the forward scan peak $\left(\mathrm{I}_{\mathrm{f}}\right)$ and the reverse scan peak $\left(\mathrm{I}_{\mathrm{r}}\right)$ in $\mathrm{CV}$ curves.

(a)

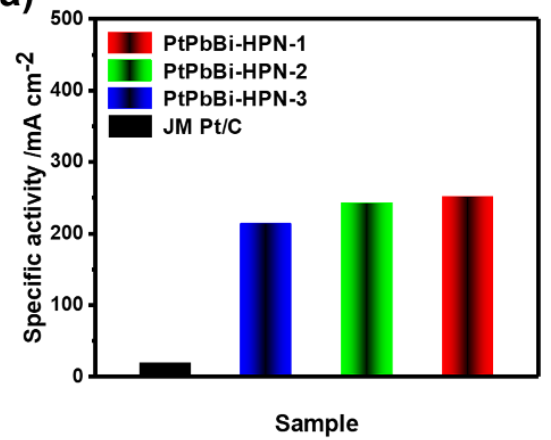

(b)

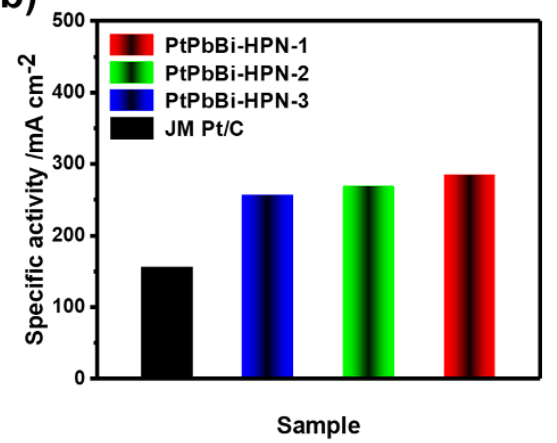

Figure S7. The histogram showing the specific activity of PtPbBi HPNs and JM Pt/C in (a) EOR (b) EGOR. 

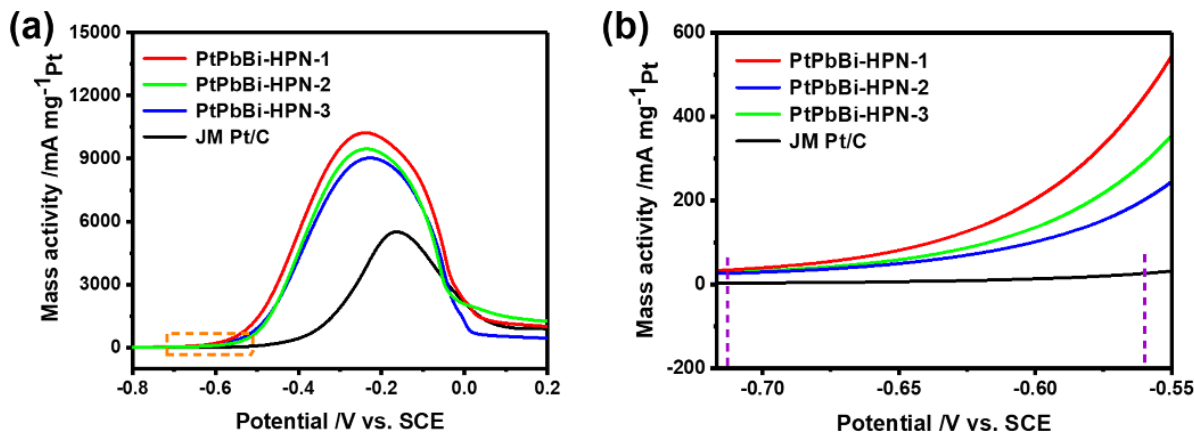

Figure S8. (a) Positive-going potential sweep curves of $\mathrm{PtPbBi} \mathrm{HPNs}$ and JM Pt/C in $\mathrm{N}_{2}$-saturated in $1 \mathrm{M} \mathrm{NaOH}$ solution containing $1 \mathrm{M}$ ethylene glycol solution at a scan rate of $50 \mathrm{mV} \mathrm{s}^{-1}$. (b) The image is enlarged from the orange squares at the inside of CV curves.

(a)

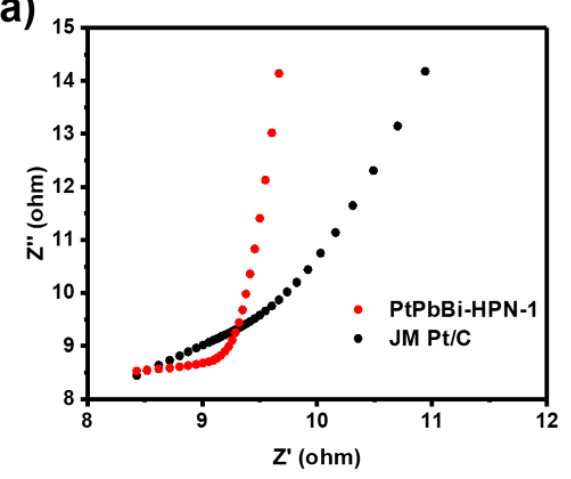

(b)

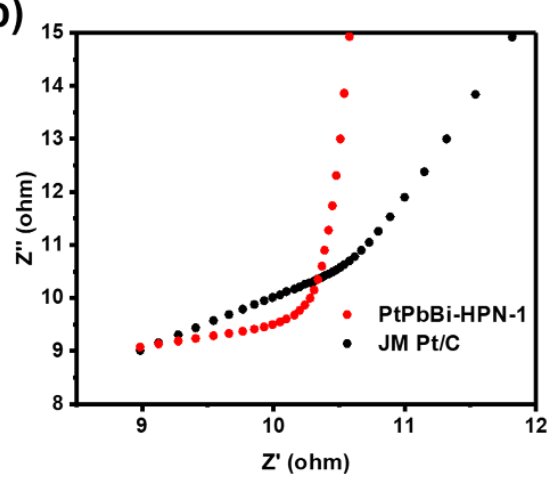

Figure S9. Nyquist plots of PtPbBi-HPN-1 and JM Pt/C in (a)1 M NaOH solution containing $1 \mathrm{M}$ ethanol solution (b)1 $\mathrm{M} \mathrm{NaOH}$ solution containing $1 \mathrm{M}$ ethylene glycol.

Table S1. Compositional analysis of PtPbBi-HPN-1 catalyst by XPS, EDS and ICPAES measurements.

\begin{tabular}{cccc}
\hline element & XPS & EDS & ICP-AES \\
\hline $\mathrm{Pt}$ & $52.3 \%$ & $57.1 \%$ & $55.7 \%$ \\
$\mathrm{~Pb}$ & $7.4 \%$ & $2.7 \%$ & $4 \%$ \\
$\mathrm{Bi}$ & $40.6 \%$ & $40.2 \%$ & $40.3 \%$ \\
\hline
\end{tabular}


Table S2. Comparison of the diffraction peak of $\mathrm{PtPbBi}-\mathrm{HPN}-1, \mathrm{PtBi} / \mathrm{C}$ and $\mathrm{PtPb} / \mathrm{C}$ for XRD pattern.

\begin{tabular}{cccc}
\hline diffraction peak & PtPbBi-HPN-1 & PtBi/C & PtPb/C \\
\hline 1 & $23.70^{\circ}$ & $23.77^{\circ}$ & $38.36^{\circ}$ \\
2 & $28.65^{\circ}$ & $28.79^{\circ}$ & $44.63^{\circ}$ \\
3 & $40.15^{\circ}$ & $40.63^{\circ}$ & $65.67^{\circ}$ \\
4 & $41.53^{\circ}$ & $41.69^{\circ}$ & $78.23^{\circ}$ \\
5 & $46.35^{\circ}$ & $46.63^{\circ}$ & $82.49^{\circ}$ \\
6 & $51.24^{\circ}$ & $51.38^{\circ}$ & - \\
7 & $55.43^{\circ}$ & $55.71^{\circ}$ & - \\
8 & $59.56^{\circ}$ & $59.64^{\circ}$ & - \\
9 & $68.04^{\circ}$ & $68.24^{\circ}$ & - \\
10 & $75.13^{\circ}$ & $75.40^{\circ}$ & - \\
11 & $81.60^{\circ}$ & $83.04^{\circ}$ & - \\
\hline
\end{tabular}


Table S3. Comparison of the mass activity of Pt-based catalysts for EOR that previously reported.

\begin{tabular}{|c|c|c|c|c|}
\hline $\begin{array}{l}\text { Sample } \\
\text { name }\end{array}$ & Condition & $\begin{array}{l}\text { Scan rate / } \\
\mathrm{mV} \cdot \mathrm{s}^{-1}\end{array}$ & $\begin{array}{c}\text { Mass } \\
\text { activity / } \\
\text { A } \text { mg }^{-1} \mathbf{P t} \\
\end{array}$ & Ref. \\
\hline PtPbBi-HPN-1 & $\begin{array}{c}1 \mathrm{M} \mathrm{NaOH}+1 \\
\text { M ethanol }\end{array}$ & 50 & 8.87 & This work \\
\hline $\begin{array}{c}\mathrm{Pt}_{2} \mathrm{Bi} \\
\text { Nanoplates }\end{array}$ & $\begin{array}{c}1 \mathrm{M} \mathrm{NaOH}+1 \\
\text { M ethanol }\end{array}$ & 50 & 5.95 & $\begin{array}{l}\text { Nano Res. } 2019,12(2) \text { : } \\
\quad 429-436\end{array}$ \\
\hline PtPdBi & $\begin{array}{c}1 \mathrm{M} \mathrm{KOH}+1 \mathrm{M} \\
\text { ethanol }\end{array}$ & 50 & 5.26 & Catalysts 2017, 7(7), 208 \\
\hline $\mathrm{Pt}_{5} \mathrm{FePd}_{2} \mathrm{NW}$ & $\begin{array}{c}1 \mathrm{M} \mathrm{KOH}+1 \mathrm{M} \\
\text { ethanol }\end{array}$ & 50 & 4.96 & $\begin{array}{c}\text { ACS Appl. Mater. } \\
\text { Interfaces } 2019,11,34 \text {, } \\
\text { 30880-30886 }\end{array}$ \\
\hline $\begin{array}{c}\mathrm{PtRh} @ \\
\mathrm{SnO}_{2} \mathrm{NWs}\end{array}$ & $\begin{array}{c}1 \mathrm{M} \mathrm{KOH}+1 \mathrm{M} \\
\text { ethanol }\end{array}$ & 50 & 3.16 & $\begin{array}{l}\text { J. Mater. Chem. A, } \\
\text { 2019,7, 27377-27382 }\end{array}$ \\
\hline $\begin{array}{c}\text { PdPtAg } \\
\text { Nanosheets }\end{array}$ & $\begin{array}{l}0.1 \mathrm{M} \mathrm{KOH} \mathrm{+} \\
0.5 \mathrm{M} \text { ethanol }\end{array}$ & 50 & 1.31 & $\begin{array}{c}\text { Angew. Chem. Int. Ed. } \\
2016,55,2753\end{array}$ \\
\hline $\begin{array}{l}\mathrm{Pt}_{54} \mathrm{Rh}_{4} \mathrm{Cu}_{42} \\
\mathrm{CNBs}\end{array}$ & $\begin{array}{c}1 \mathrm{M} \mathrm{KOH}+1 \\
\text { M ethanol }\end{array}$ & 50 & 4.09 & $\begin{array}{l}\text { Adv. Energy Mater. } \\
\text { 2018, 8, } 1801326\end{array}$ \\
\hline $\begin{array}{c}\text { PdPtAg } \\
\text { nanosheets }\end{array}$ & $\begin{array}{c}1 \mathrm{M} \mathrm{KOH}+0.5 \\
\text { M ethanol }\end{array}$ & 50 & 1.40 & $\begin{array}{l}\text { Angew. Chem. Int. Ed. } \\
2016,55,2753-2758 .\end{array}$ \\
\hline NiAuPt -NGs & $\begin{array}{c}0.5 \mathrm{M} \mathrm{KOH} \mathrm{+1} \\
\mathrm{M} \text { ethanol }\end{array}$ & 50 & 4.94 & $\begin{array}{c}\text { ACS Catal. 2015, 5, } \\
\text { 1371-1380. }\end{array}$ \\
\hline $\mathrm{PtPd}_{3} \mathrm{Ag}_{5} / \mathrm{C}$ & $\begin{array}{c}1 \mathrm{M} \mathrm{KOH}+1 \\
\text { M ethanol }\end{array}$ & 50 & 4.50 & $\begin{array}{c}\text { Electrochim. Acta } 2017, \\
236,72-81\end{array}$ \\
\hline $\mathrm{Pt}_{2} \mathrm{Fe} \mathrm{NCs}$ & $\begin{array}{c}1 \mathrm{M} \mathrm{KOH}+1 \\
\text { M ethanol }\end{array}$ & 50 & 3.96 & $\begin{array}{c}\text { ChemCatChem 2018, } 10 \text {, } \\
2195-2199 .\end{array}$ \\
\hline $\begin{array}{l}\mathrm{Pt}_{3} \mathrm{Rh}_{1} \mathrm{Ni}_{2-} \\
\text { ANAs }\end{array}$ & $\begin{array}{c}1 \mathrm{M} \mathrm{NaOH}+1 \\
\text { M ethanol }\end{array}$ & 50 & 1.39 & $\begin{array}{l}\text { Nano Res. 2017, 10, } \\
\text { 3324-3332. }\end{array}$ \\
\hline
\end{tabular}


Table S4. Comparison of the mass activity and stability of Pt-based catalysts for EGOR that previously reported.

\begin{tabular}{|c|c|c|c|c|}
\hline $\begin{array}{l}\text { Sample } \\
\text { name }\end{array}$ & Condition & $\begin{array}{l}\text { Scan rate / } \\
\mathrm{mV} \cdot \mathrm{s}^{-1}\end{array}$ & $\begin{array}{c}\text { Mass } \\
\text { activity / } \\
{\text { A } \text { mg }^{-1} \mathbf{P t}}\end{array}$ & Ref. \\
\hline PtPbBi-HPN-1 & $\begin{array}{l}1 \mathrm{M} \mathrm{NaOH} \\
+1 \mathrm{MEG}\end{array}$ & 50 & 12.22 & This work \\
\hline $\mathrm{Pt}_{4} \mathrm{Co}$ Nanocube & $\begin{array}{c}1 \mathrm{M} \mathrm{KOH}+ \\
1 \mathrm{M} \mathrm{EG}\end{array}$ & 50 & 9.34 & $\begin{array}{c}\text { Nanoscale, } 2020,12, \\
9842-9848\end{array}$ \\
\hline $\mathrm{Pt}_{3} \mathrm{Ni} \mathrm{SNWs}$ & $\begin{array}{c}1 \mathrm{M} \mathrm{KOH}+ \\
1 \mathrm{M} \mathrm{EG}\end{array}$ & 50 & 4.88 & $\begin{array}{c}\text { Nanoscale, 2019,11, } \\
4831-4836\end{array}$ \\
\hline $\mathrm{Pt}_{4} \mathrm{Rh}-\mathrm{SNCs}$ & $\begin{array}{l}1 \mathrm{M} \mathrm{KOH} \\
+1 \mathrm{M} \mathrm{EG}\end{array}$ & 50 & 5.12 & $\begin{array}{l}\text { J. Mater. Chem. A, } \\
\text { 2019,7, 7891-7896 }\end{array}$ \\
\hline $\mathrm{Pt}_{1} \mathrm{Au}_{1} \mathrm{Ag}_{1}$ & $\begin{array}{l}1 \mathrm{M} \mathrm{KOH} \\
+1 \mathrm{MEG}\end{array}$ & 50 & 6.35 & $\begin{array}{l}\text { J. Power Sources } \\
2018,384,42-47 .\end{array}$ \\
\hline $\begin{array}{c}\mathrm{Pt}-\mathrm{Co} \\
\text { EDNC/C }\end{array}$ & $\begin{array}{c}1 \mathrm{M} \mathrm{KOH} \\
+0.1 \mathrm{M} \mathrm{EG}\end{array}$ & 50 & 2.5 & $\begin{array}{c}\text { Appl. Catal., B: } \\
\text { Environ., } \\
\text { 2019, 258, } 117951 .\end{array}$ \\
\hline $\mathrm{Pt}_{1} \mathrm{Cu}_{1}$ & $\begin{array}{c}1 \mathrm{M} \mathrm{KOH}+ \\
1 \mathrm{M} \mathrm{EG}\end{array}$ & 50 & 2.15 & $\begin{array}{l}\text { J. Colloid Interf. Sci., } \\
\text { 2019, 551, 81-88. }\end{array}$ \\
\hline $\mathrm{Pt}-\mathrm{Co}$ EDNC/C & $\begin{array}{c}1 \mathrm{M} \mathrm{KOH} \\
+0.1 \mathrm{M} \mathrm{EG}\end{array}$ & 50 & 2.5 & $\begin{array}{c}\text { Appl. Catal., B: } \\
\text { Environ., 2019, 258, } \\
117951 .\end{array}$ \\
\hline PtRu alloy & $\begin{array}{l}1 \mathrm{M} \mathrm{KOH} \\
+1 \mathrm{MEG}\end{array}$ & 50 & 3.05 & $\begin{array}{c}\text { Int. J. Hydrogen } \\
\text { Energy, 2017, 42, } \\
\text { 20720-20728. }\end{array}$ \\
\hline
\end{tabular}

